\title{
Investigation of the neoclassical ambipolar electric field in ion-root plasmas on W7-X
} \author{
W7-X Team \\ ${ }^{1}$ Princeton Plasma Physics Laboratory, USA \\ ${ }^{2}$ Max-Planck-Institut für Plasmaphysik, Germany \\ ${ }^{3}$ Forschungszentrum Jülich, Germany \\ ${ }^{4}$ Laboratorio Nacional de Fusión, CIEMAT, Spain \\ ${ }^{5}$ Auburn University, USA \\ ${ }^{6}$ National Institute for Fusion Science, Japan \\ ${ }^{7}$ University of Maryland, USA \\ ${ }^{8}$ Laboratory for Plasma Physics, LPP-ERM/KMS, Brussels, Belgium
}

N. Pablant ${ }^{1}$, A. Langenberg ${ }^{2}$, A. Alonso ${ }^{4}$, J. Baldzuhn², C.D. Beidler ${ }^{2}$, S. Bozhenkov ${ }^{2}$, R. Burhenn $^{2}$, K.J. Brunner ${ }^{2}$, A. Dinklage ${ }^{2}$, G. Fuchert ${ }^{2}$, O. Ford ${ }^{2}$, D.A. Gates ${ }^{1}$, J. Geiger ${ }^{2}$,

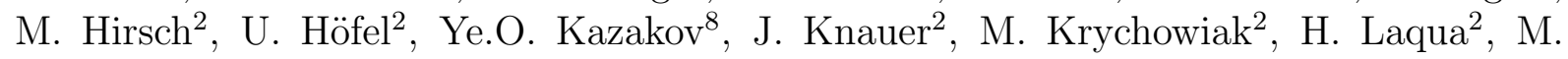
Landreman $^{7}$, S. Lazerson ${ }^{1}$, H. Maaßberg ${ }^{2}$, O. Marchuck ${ }^{3}$, A. Mollen ${ }^{2}$, E. Pasch ${ }^{2}$, A. Pavone $^{2}$, S. Satake ${ }^{6}$, T. Schröder ${ }^{2}$, H.M. Smith ${ }^{2}$, J. Svensson ${ }^{2}$, P. Traverso ${ }^{5}$, Y. Turkin ${ }^{2}$, J.L. Velasco ${ }^{4}$, A. von Stechow ${ }^{2}$, F. Warmer ${ }^{2}$, G. Weir ${ }^{2}$, R.C. Wolf ${ }^{2}$, D. Zhang ${ }^{2}$ and the

Corresponding Author: npablant@pppl.gov

\begin{abstract}
:
The role of the radial electric field in high-performance ion-root plasmas on Wendelstein 7-X (W7-X) is examined and compared with neoclassical predictions. The W7-X stellarator is the world's first large-scale optimized stellarator. One of the important targets chosen for optimization during the W7-X design process was the reduction of core neoclassical heat transport. This optimization was targeted for reactor-relevant high-density plasmas with $T_{e} \approx T_{i}$ in which the neoclassical ambipolar radial electric field is expected to be negative throughout the plasmas core.

Measurements of the core radial electric field $\left(E_{r}\right)$ have confirmed that ion-root conditions (negative $E_{r}$ in the plasma core) have been achieved in W7-X with high-density plasmas and central ECRH. These measured $E_{r}$ profiles agree well with the neoclassical ambipolar $E_{r}$ predicted by the code SFINCS. This good agreement provides confidence in the validity of neoclassical calculations in high-density ion-root conditions, and enables initial studies on the role of neoclassical transport in the optimized high-density regime of W7-X.

Profile measurements of electron temperature $\left(T_{e}\right)$, ion temperature $\left(T_{i}\right)$ and electron density $\left(n_{e}\right)$ along with approximations for the average value of $Z_{e f f}$ have been used as inputs to the SFINCS code to calculate the ambipolar $E_{r}$ profile along with neoclassical ion and electron energy flux profiles $\left(Q_{N C i}, Q_{N C e}\right)$. Finally the total experimental energy input to the plasma from ECRH heating has been compared to the neoclassical energy fluxes to
\end{abstract}


provide a first estimate for the fraction of transport that can be attributed to neoclassical processes in reactor-relevant high-density ion-root conditions.

\section{Introduction}

The Wendelstein 7-X (W7-X) stellarator is the world's first large-scale optimized stellarator [1, 2]. One of the important targets chosen for optimization during the W7-X design process was the reduction of core neoclassical (NC) energy transport [3]. This optimization was targeted for reactor relevant [4] ECRH (electron cyclotron resonance heating) heated plasmas with $T_{e} \approx T_{i}$ in which the neoclassical ambipolar radial electric field is expected to be negative throughout the plasma core. An important step in evaluating the effectiveness of the NC optimization is to demonstrate that these plasma conditions are achievable experimentally and validate the use of our current neoclassical codes with the experimental plasma profiles. Of particular interest for validation is the radial electric field, which is expected to arise only from neoclassical effects.

In stellarator plasmas the neoclassical particle fluxes are not intrinsically ambipolar; this leads to the generation of a radial electric field profile $\left(E_{r}\right)$ that provides for ambipolarity across the plasma radius. Furthermore, in the absence of significant fast-ion losses, the radial electric field is thought to be fully described by neoclassical theory as turbulent particle transport is expected to be intrinsically ambipolar to leading order [5, 6]. The details of the $E_{r}$ profile are expected to have a strong effect on the NC heat, particle, and impurity fluxes as well as the bootstrap current [7, 8].

Radial electric fields and the comparison between $\mathrm{NC}$ theory and experiments have been studied on other many other stellarator devices [9, 10] including LHD [11, 12], W7AS [13, 14], TJ-II [15], HSX [16, 17], CHS [18, 19] and Heliotron-J [20]. The experimental uncertainties and level of quantitative agreement varies between these studies, however overall they all point towards the validity of neoclassical theory in describing the ambipolar radial electric field. The work described in the current paper is important in extending these types of comparisons to a neoclassically optimized stellarator (W7-X) over a wide range of plasma conditions.

For the W7-X geometry, the NC ambipolar electric field is expected to be positive when $T_{e} \gg T_{i}$ (known as the electron-root) and negative when $T_{e} \approx T_{i}$ (known as the ion-root). The main source of plasma heating is through ECRH, which only provides energy to the electrons. To achieve equilibration of the ion and electron temperatures requires operation at high densities (around $1 \times 10^{20} \mathrm{~m}^{-3}$ ) to provide sufficient collisional coupling.

Measurements of the core radial electric field $\left(E_{r}\right)$ have confirmed that ion-root conditions have been achieved on W7-X. In this paper these measurements will be presented and compared with neoclassical calculations, providing a first experimental validation of $\mathrm{NC}$ theory in W7-X for high-density ion-root conditions. These conditions are close to the target point for the W7-X optimization, except for being at a lower total plasma pressure due to the modest amount of available heating power. 


\section{Observation of ion-root in pellet-fueled plasmas}

The most clear observations of ion-root conditions in W7-X have been in plasmas which utilize cryogenic hydrogen pellet injection 21] and central ECRH heating, see Fig, 1 22, 23. During the Op1.2 experimental campaign[24] the available pellet injection system was limited to an operational time of $1 \mathrm{~s}$. This limitation results in a highly transient high density phase in the plasma evolution. While the plasma conditions are non-stationary, the radial electric field is expected to respond nearly immediately (faster than the transport time scale) to changes in the temperature and density profile[25], and therefore its neoclassical analysis is appropriate in this context. It should be noted that the program that will be the focus of the subsequent analysis is not unique, and that many plasmas with similar time-histories have been produced at W7-X[23].

Program 20171207.006 starts with a low density plasma with 3.0 MW of central ECRH heating. From $1.2 \mathrm{~s}$ to $1.8 \mathrm{~s}$ hydrogen pellets are injected into the plasma with a frequency of $30 \mathrm{~Hz}$. After the start of the pellet injection additional central ECRH power is added to bring the total injected power to 5.0 MW. During pellet injection the central electron density rises to a value of $0.9 \times 10^{20} \mathrm{~m}^{-3}$ and also becomes peaked, as can be seen by the separation of the central density from the line-integrated density in Fig 1 $1 \mathrm{~b}$. Immediately after pellet injection the electron temperature falls to meet the ion temperature; after reaching equilibration both the $T_{e}$ and $T_{i}$ profiles begin to increase together, ultimately reaching an equilibrated core temperature of $T_{i}=3.4 \mathrm{keV}, T_{e}=3.9 \mathrm{keV}$ at $2.2 \mathrm{~s}$. During this period of collisionally coupled heating the stored energy and plasma energy confinement time increase dramatically up to values of $1.1 \mathrm{MJ}$ and $0.2 \mathrm{~s}$ respectively[26].

The evolution of the radial electric field profiles from this program, which are inferred from the X-Ray Imaging Crystal Spectrometer (XICS) diagnostic 27, 28, are shown in Fig,2a. In this figure the $E_{r}$ profiles are plotted against time and the normalized minor radius, which is defined as $\rho \equiv \sqrt{\psi / \psi_{\text {edge }}}$ where $\psi$ is the toroidal
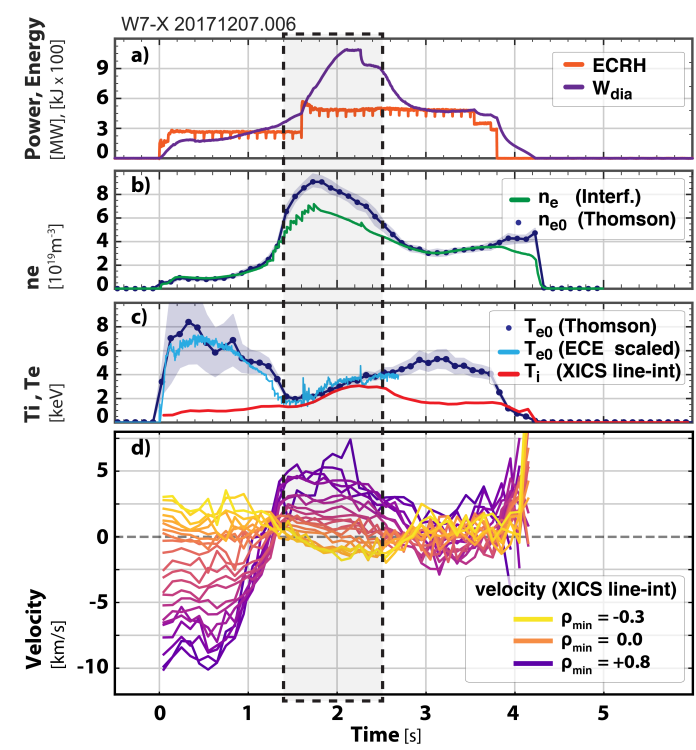

FIG. 1: Time traces for program 20171207.006. Cryogenic hydrogen pellets are injected from $1.2 \mathrm{~s}$ to $1.8 \mathrm{~s}$. The time when the radial electric field is fully within the ion-root is highlighted with the dashed box. Electron temperature and density taken from the central Thomson channels. Fig. (d): Line-integrated plasma velocity for several XICS sightlines; purple and yellow lines represent views above and below the magnetic axis respectively. The change in the direction of the plasma velocity can be clearly seen in these non-inverted measurements. flux enclosed by a given flux surface. These measurements confirm the existence of a negative radial electric field in the plasma core (ion-root conditions) during the high-density pellet-fueled phase of the program where $T_{e} \approx T_{i}$. During beginning and ending phases of this program, where the plasma den- 
sity is lower and $T_{e} \gg T_{i}$, a positive core radial electric field is observed. Details of the diagnostic method used to produce these profiles are provided in Section 5 .

The $E_{r}$ profiles for selected times in the discharge are shown in Fig, 3 along with the temperature and density profiles. In these figures the error bars represent only the statistical error due to photon statistics, and do not include any systematic errors. It is important to note that the kinks seen in the $E_{r}$ profiles are due to over-fitting (undersmoothing) during the $E_{r}$ tomographic inversion and not due to the actual profile shape. The presentation of these profiles without additional smoothing has been chosen in order to illustrate the available resolution of the measurements and insure that structure is not artificially imposed through smoothing constraints.

The ion-root phase of this program, in which the radial electric field is negative all the way into the plasma core, exists between $1.40 \mathrm{~s}$ and $2.50 \mathrm{~s}$, as seen in Fig. $2 \mathrm{a}$ and highlighted in Fig.1. The precise timing of the ion-root phase has been determined using an analysis completed using a $20 \mathrm{~ms}$ integration time (not shown in the current paper), and is expected to be accurate to within this resolution. At the point in which the full ion-root condition develops, at $1.40 \mathrm{~s}$, the following plasma parameters are observed: $T_{e 0}=2.25 \mathrm{keV}, T_{i 0}=1.4 \mathrm{keV}, \bar{n}_{e}=0.45 \times 10^{20} \mathrm{~m}^{-3}$ and $P_{E C R H}=2.7 \mathrm{MW}$. At the start of the transition out of the ion root, at $2.50 \mathrm{~s}$, the following plasma parameters are observed: $T_{e 0}=4.4 \mathrm{keV}, T_{i 0}=3.3 \mathrm{keV}, \bar{n}_{e}=0.45 \times 10^{20} \mathrm{~m}^{-3}$ and $P_{E C R H}=5.0 \mathrm{MW}$. Central electron densities at the transition are approximately $0.6 \times 10^{20} \mathrm{~m}^{-3}$ indicating moderate peaking of the density profile (see Fig.3b). Measurements of the electron temperature and density profiles are taken from the Thomson scattering diagnostic [29], while the lineintegrated electron density is taken from the interferometer[30]. At both ends of the ion-root phase we find a similar value of the electron density as well as a similar difference between the absolute ion and electron temperatures, approx $1.0 \mathrm{keV}$ as seen in Fig.1.

Leading up to full development of the ion-root plasma at $1.40 \mathrm{~s}$, it can be observed that an ion-root region develops in the outer portion of the plasmas and expands inward. This change in $E_{r}$ occurs over a time period of around $0.5 \mathrm{~s}$, and can be seen to follow the evolution of the central temperature and density values. Similar behavior is seen as the plasma transitions out of the ion root after $2.50 \mathrm{~s}$ and develops an expanding region of positive radial electric field in the core. The evolution of the $E_{r}$ inversion radius is highlighted in Fig 2 with dashed lines. The detailed time dependence of changes to the $E_{r}$ profile can be more clearly observed by looking directly at the line-integrated plasma flow measurements shown in Fig.1 d. Within the resolution of the available measurements, the electron temperature and plasma rotation appear to change simultaneously, which is consistent with the neoclassical understanding of $E_{r}$. These observations of changes in the radial electric field profile in response to changing plasma conditions are in line with studies of the radial electric field in electron-root plasmas at different input powers as seen in previous work on W7-X[28] and LHD[12]. 


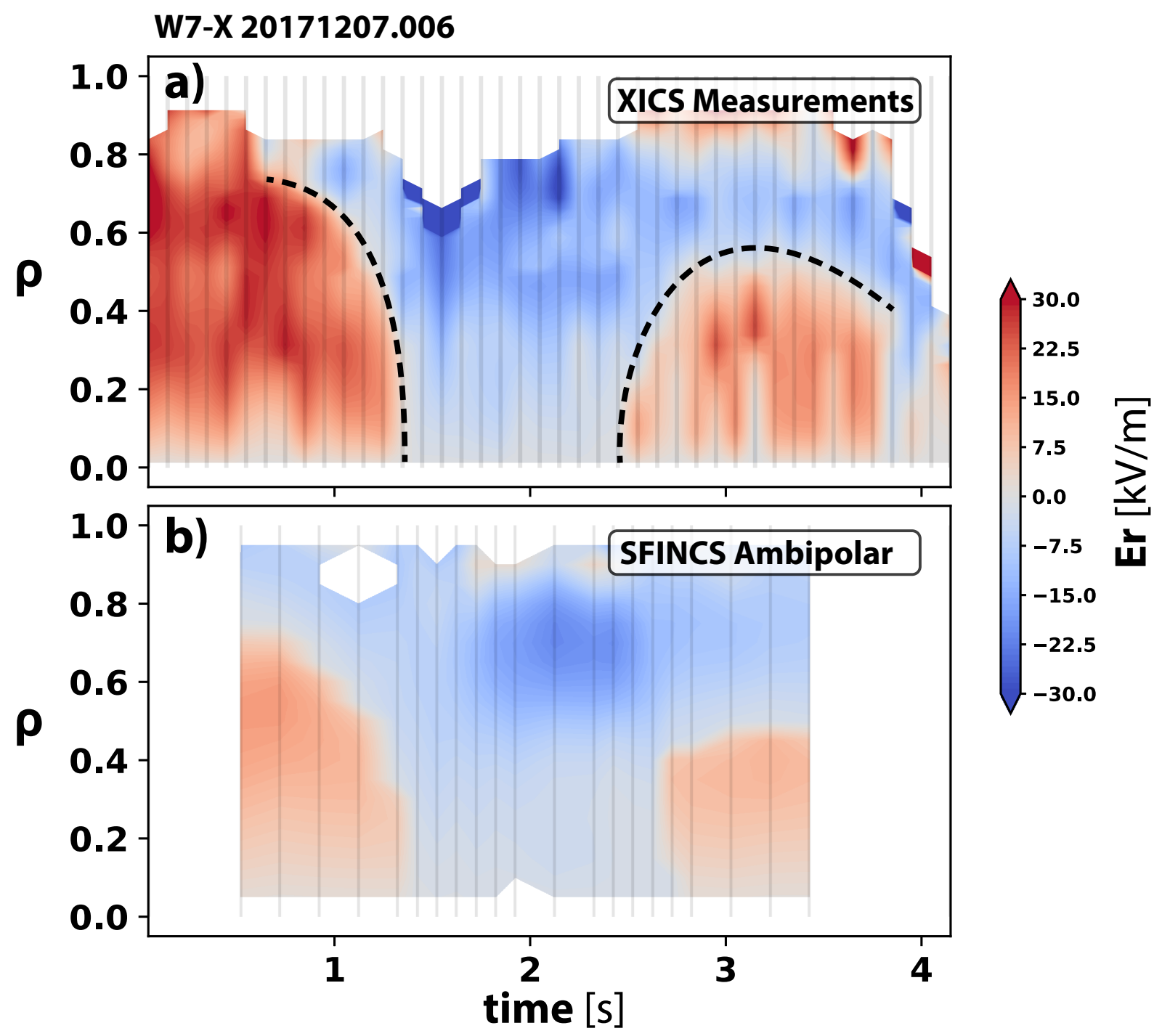

FIG. 2: Radial electric field: a) as inferred from XICS measurements, b) ambipolar radial electric field calculated by SFINCS. Both plots use the same color scale. For XICS measurements raw data has been binned prior to inversion to provide $100 \mathrm{~ms}$ time resolution and $3 \mathrm{~cm}$ spatial resolution. Vertical lines denote actual measurement times (center of integration window); color between lines is interpolated. Shortly after $1.5 \mathrm{~s}$ a strong peaking in the Ar ${ }^{16+}$ emissivity profile results in a limited measurable profile extent; radii with insufficient signal for valid measurements are left white. The dashed lines indicate the $E_{r}$ inversion radius at which the radial electric field changes sign. For the SFINCS results the vertical lines denote the times at which calculations were performed, and correspond to the time of the Thomson Scattering measurement (the nearest XICS interval was used). 


\section{Neoclassical comparison}

The neoclassical (NC) ambipolar radial electric field profile can be found by solving the drift-kinetic equation given a set of temperature and density profiles and a plasma equilibrium and scanning $E_{r}$ for the ambipolar solution [31, 32. The results of such a calculation, using the SFInCs 32 code, are shown in Fig. $2 \mathrm{~b}$ and Fig. 3 . The contour plot shown in Fig $2 \mathrm{~b}$ is made from profiles of the neoclassical ambipolar Er calculated for 21 selected times between $0.5 \mathrm{~s}$ and $3.5 \mathrm{~s}$. Three of these Er profiles are shown in comparison to the XICS measurements in Fig,3, along with the temperature and density profiles used as inputs to the SFINCS; the electron temperature and density profiles are taken from the Thomson scattering diagnostic 29], and the ion temperature is taken from the XICS diagnostic. Here the electron density profiles have been scaled to match the line-integrated measurements from the interferometer 33] resulting in a scaling factor of approximately 1.1.

a) $2.20 \mathrm{~s}$

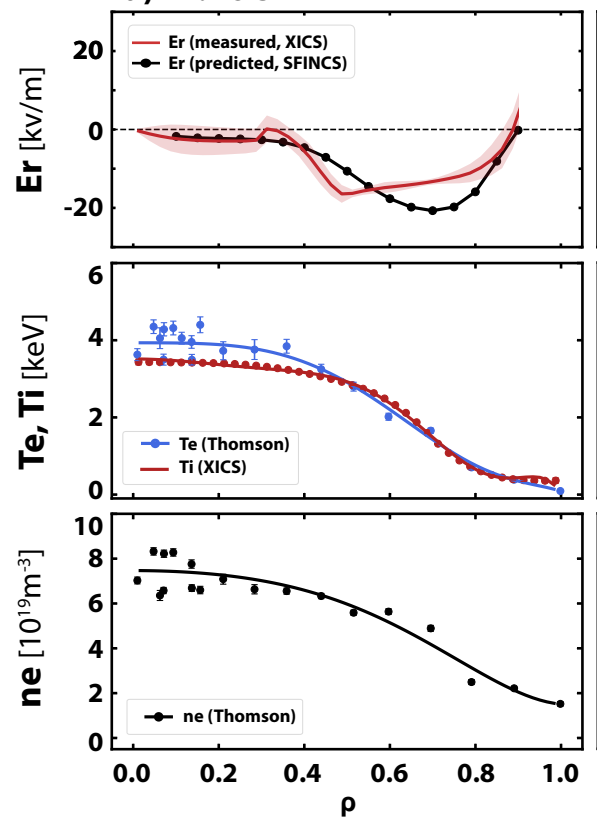

b) $2.50 \mathrm{~s}$
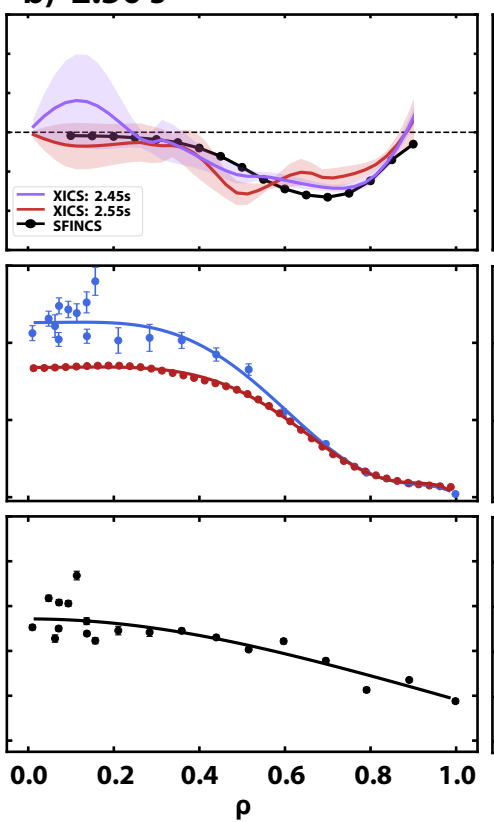

c) $3.40 \mathrm{~s}$
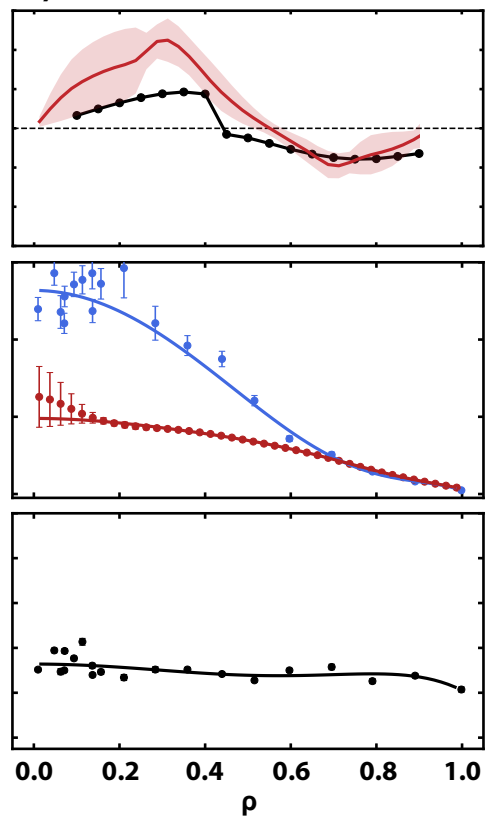

FIG. 3: Comparison of the neoclassical ambipolar radial electric field, calculated using the SFIncs code, with the measured profiles from the XICS diagnostic. Three times are shown which highlight different plasmas conditions found within this discharge. $2.20 \mathrm{~s}$ : Time of peak stored energy, when the plasma is fully within the ion root. $2.50 \mathrm{~s}$ : Time of transition when the electron root first reappears in the plasma core $\left(E_{r}\right.$ profiles from XICS are shown for two consecutive timeslices showing the appearance of the electron root). $3.40 \mathrm{~s}$ : Steady-state period far from the pellet injection where the plasma is in the electron root out to $\rho \approx 0.5$. The shaded region around the measured $E_{r}$ profiles and the error bars around the temperature and density points correspond to the one sigma error due to photon statistics and are found using a Monte-Carlo procedure. Systematic errors are not shown and may be significantly larger.

This set of $\mathrm{NC}$ calculations was done with an approximation of $Z_{\text {eff }}=1$ and used a vacuum vMEc 34] equilibrium (zero plasma pressure). The experimental line-of-sight average $Z_{\text {eff }}$ values during this experiment were approximately in the range of $2-3 \pm 30 \%$ 


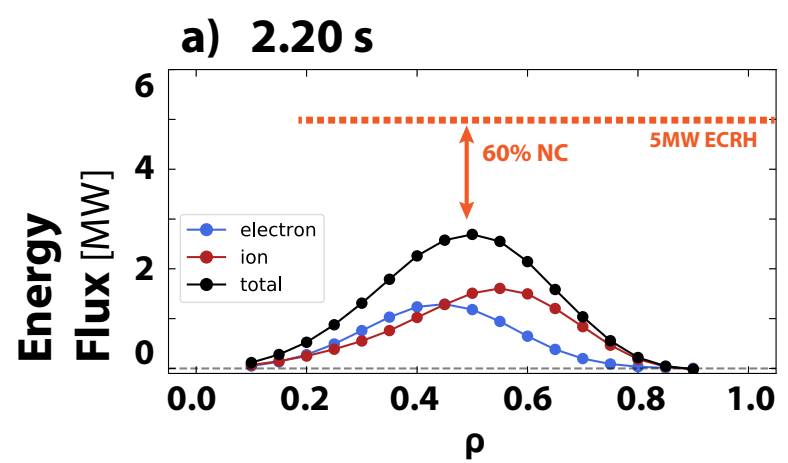

b) $3.40 \mathrm{~s}$

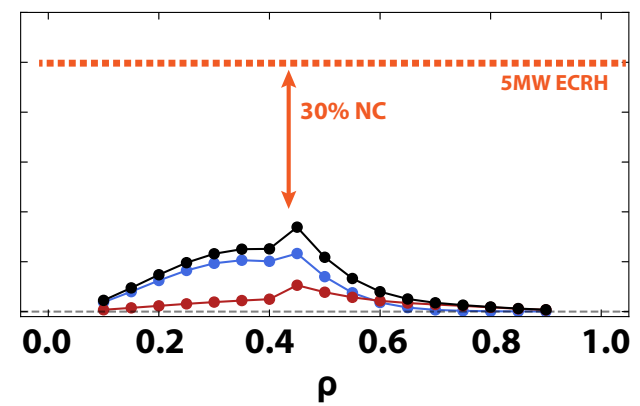

FIG. 4: Neoclassical energy flux (calculated by SFINCS) as compared to the total input power from ECRH. These calculations represent the total NC energy flux through a given flux surface.

as measured using visible Bremsstrahlung radiation[35]. However, measurements of $Z_{\text {eff }}$ profiles are not currently available, and the core value could be substantially different. The choice for assuming zero plasma pressure was made to simplify calculations in that a single equilibrium could be used throughout the analysis. The calculations of $E_{r}$ and energy fluxes are largely insensitive to both $Z_{\text {eff }}$ and plasma pressure (see Fig.8), justifying the use of these approximations. A detailed study on the sensitivity to these approximations, along with a study on the profile sensitivity, is discussed in Section 6 .

A comparison of the neoclassical calculations and measured values of $E_{r}$ from XICS are shown in both figures Fig 2 and Fig. 3 . This comparison show generally good agreement in both the profile shape and in time evolution as the XICS measurements, including the evolution of the inversion radius. The magnitude of the Er is also in good agreement and at all times within about a factor of two, and within error bars in many cases. Importantly, this agreement is seen throughout the discharge even as the plasma profiles and heating power vary considerably, capturing a significant portion of the W7-X operating space. Agreement is seen both during the ion-root phase and the electron-root phases. This agreement provides confidence in the neoclassical calculations, and serves as a validation of NC particle and heat flux predictions over a wide range of W7-X plasma conditions, and in particular in the high-density ion-root regime.

The differences seen between the measurements and the NC expectations in Fig 3 are up to a factor of two in magnitude, especially during the electron-root. However, given the sizable random and systematic errors in the profile measurements, this can still be seen as being within reasonable agreement at this level of profile accuracy. A discussion on sources of uncertainty and possible explanations for disagreements in the magnitude of $E_{r}$ can be found in Section 7 .

The comparison between calculated and measured $E_{r}$, keeping in mind uncertainties in the measured $E_{r}$ profile, provides confidence in the validity of neoclassical calculations. With this in mind an initial investigation into the role of neoclassical transport in highdensity ion-root plasmas is shown in Fig 4 . Here the neoclassical ion and electron energyflux profiles $\left(Q_{N C i}, Q_{N C e}\right)$, integrated over the flux surface area, are shown for two different times in the plasmas and compared to the total heating power from ECRH. In both the 
ion-root phase and electron-root phase the electron energy flux is fairly similar, however the ion energy flux can be seen to be much more significant during the ion-root phase. This change is significant enough that ions, rather than electrons, become the dominant NC loss channel.

From the simple comparison between input power and total $\mathrm{NC}$ energy flux it can be seen that during the ion-root phase of this discharge approximately $60 \%$ of the core transport can be attributed to neoclassical energy flux, while in the electron-root phase at the end of the discharge less than $30 \%$ of core transport can be attributed to NC processes. The difference between the NC energy flux and the input power (the anomalous transport) is likely attributable to turbulent transport. As expected, in the outer portion of the plasma NC transport plays a minor role and energy loss is dominated by other sources as turbulent transport, radiation or charge exchange losses.

The plasma stored energy and global confinement at the two times shown in Fig. 4 have the following values: at $2.2 \mathrm{~s} W_{d i a}=1.1 \mathrm{MJ}$ and $\tau_{E}=0.2 \mathrm{~s}$ while at $3.4 \mathrm{~s} W_{d i a}=0.5 \mathrm{MJ}$ and $\tau_{E}=0.1 \mathrm{~s}$, where $W_{d i a}$ is measured by a diamagnetic loop[36] and $\tau_{E}=W_{d i a} /\left(P_{E C R H}-\right.$ $\left.d W_{\text {dia }} / d t\right)$. While the plasma heating is the same at both times, the plasma confinement is much better at $2.2 \mathrm{~s}$, even though the neoclassical energy flux has more than doubled. From these observations it becomes immediately clear that the level of anomalous transport has changed dramatically.

A more detailed power-balance analysis for a similar pellet fueled plasma can be found in Ref. 23. This reference also provides in depth discussion on the role of neoclassical transport in both the ion and electron transport channels. A detailed investigation on changes in the turbulent transport, along with measurements of plasma fluctuations and theoretical considerations, is underway and will be reported in a
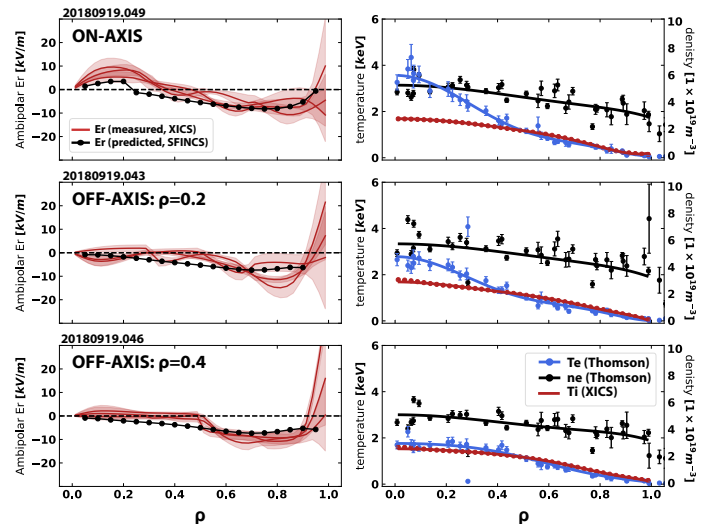

FIG. 5: Effect of electron temperature profile shaping through off-axis heating on $E_{r}$ profiles. In the on-axis case $3.5 \mathrm{MW}$ of ECRH heating is deposited at the plasma center, in the two off-axis cases the same amount of ECRH heating is deposited at a normalized minor radius of $\rho=0.2$ and $\rho=0.4$. For the XICS measurements the results from three consecutive measurements with $100 \mathrm{~ms}$ time integration are shown, providing another view into the errors in the measurement and subsequent inversion process. future publication[24].

Finally, the agreement between NC predictions and measurements of $E_{r}$ also supports the conclusion that turbulent transport in W7$\mathrm{X}$ is intrinsically ambipolar. While it is observed that core turbulent transport changes dramatically during different parts this discharge, in all cases the measured $E_{r}$ profiles closely match neoclassical expectations. Also it can be seen in Fig 1 that changes in the measured plasma velocity closely follow the changes in the profile evolution, as would be expected for a purely $\mathrm{NC} E_{r}$. 


\section{Additional $E_{r}$ comparisons}

In this section several additional comparisons between neoclassical and measured $E_{r}$ profiles are shown. These studies serve to show the dependence of $E_{r}$ to magnetic configuration, temperature profile shaping, and the used of neutral beam injection.

The ECRH system at W7-X is steerable allowing power to be deposited off axis. The primary effect of changing the ECRH deposition profile is a modification of the central electron temperature; other plasma parameters, such as the ion temperature and electron density remain constant. This capability provides an ideal method to examine the dependence of the $E_{r}$ profile and the ion-root transition with a simple dependence on the $T_{e}$ profile. A set of experiments to examine this dependence is shown in Fig.5. Three similar plasmas in the standard configuration (EIM) are studied in which the deposition location of the $\mathrm{ERCH}$ power is changed from on-axis to a normalized minor radius of $\rho=0.2$ and finally to $\rho=0.4$. Only the case with on-axis heating is predicted to have an electron-root solution in the core by neoclassical calculations; this agrees well with the $E_{r}$ measurements from the XICS diagnos-

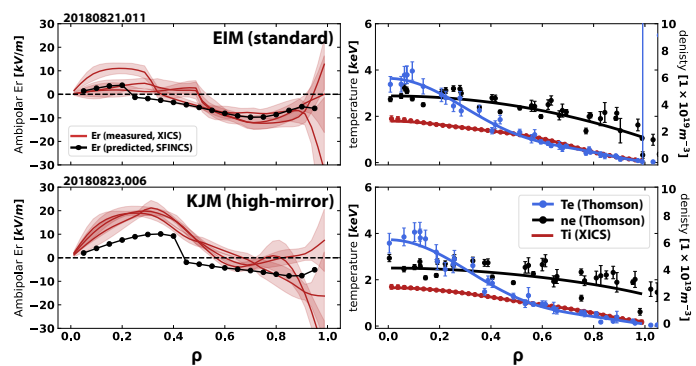

FIG. 6: Radial electric field profile dependence on changes in magnetic configuration. Here plasmas with two different magnetic configurations are shown: top plots show the standard configuration (EIM), bottom plots show the high-mirror configuration (KJM). While the temperature and density profiles in the two cases are closely matched, a significant change in the $E_{r}$ profile is seen in both the neoclassical calculation and the XICS measurements. As in the previous figure, three consecutive XICS measurements are shown with $100 \mathrm{~ms}$ integration. tics which show a core-electron root region only in this case. The existence of an ambipolar electron-root solution at a particular radius in the SFINCS calculations is quite sensitive to the electron temperature gradient, which is consistent with these observations. Uncertainties in the $T_{e}$ gradients may be one explanation for the small difference in the $E_{r}$ inversion radius. In all three cases good agreement in the profile shape and magnitude is observed.

Another study that has been carried out is to show the dependence of the radial electric field profile on the magnetic geometry. Two plasma discharges have been chosen that have different magnetic configurations and closely matched temperature and density profiles. Both cases also have very similar heating of $3.5 \mathrm{MW}$ of central ECRH power. The $E_{r}$ profiles, both from the XICS diagnostic and SFInCS neoclassical calculations, are shown in Fig.6. A very clear change in the radial electric field profiles can be seen: in the standard configuration case only a very small region in the core has an electron-root solution, while in the high-mirror case a strong electron-root solution is found over a large portion of the plasma, up to half of the minor radius. The trend in the $E_{r}$ profile is well matched between the measured profiles and the NC predictions, including the increase in strength of the electric field and the expansion of the electron root region. In the high-mirror configuration case a discrepancy in the absolute magnitude of about a factor of two between measurements and prediction can be seen (see discussion in Section 7). 
In addition to ECRH heating, W7-X also has available $3.5 \mathrm{MW}$ of neutral beam injection (NBI) heating [37]. NBI provides not only ion and electron heating, but also a source of core fueling. In plasmas sustained only with neutral beam heating a clear ion-root signature is also observed. The evolution of the measured $E_{r}$ profiles in a NBI heated discharge (20180919.033) is shown in Fig.7. At the beginning of this discharge $2 \mathrm{MW}$ of central ECRH heating is used to provide a background plasma into which NBI can be used. At $1.5 \mathrm{~s}$ the ECRH heating is turned off, and heating is switched to $3.5 \mathrm{MW}$ of NBI. The ECRH phase is a typical moderate density W7-X plasma $\left(n_{e}=0.6 \times 10^{20} \mathrm{~m}^{-3}, T_{e}>T_{i}\right)$ with a core region of positive radial electric field (electronroot).

Immediately after the switch to NBI heating (within $100 \mathrm{~ms}$ ) the radial electric field is found to transition from having positive value in the core (typical of low/moderate density ECRH plasmas) to having negative values across the plasmas radius (ion root). During the NBI phase the density becomes peaked, and $T_{i}$ is found to be slightly larger than $T_{e}$. Again good agreement is seen between neoclassical prediction and XICS measurements. It is important to note that the neoclassical calculations per-
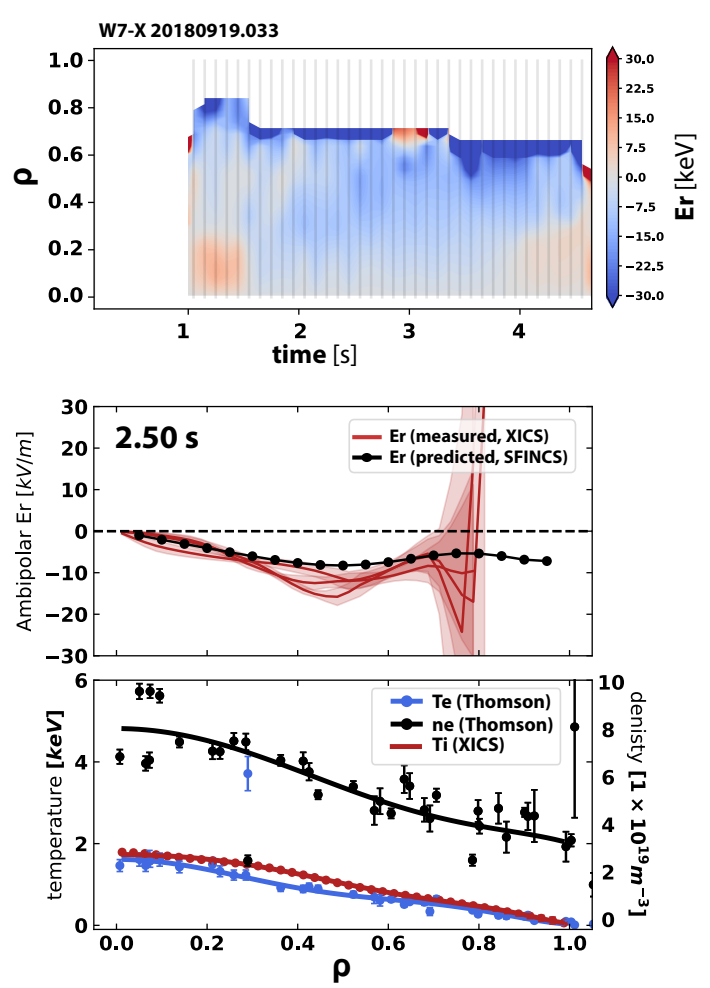

FIG. 7: Top figure: Measured radial electric field evolution for an NBI heated discharge from the XICS diagnostic. Heating is switched from ECRH to NBI at $1.5 \mathrm{~s}$. Middle figure: Profiles of the radial electric field during NBI heating from XICS measurements and SFINCS predictions. Bottom figure: plasma profiles at $2.5 \mathrm{~s}$ used for neoclassical calculations of the ambipolar radial electric field.

formed here did not consider the effect of the fast-ion population or radial currents due to fast ion-loss in determining the ambipolar $E_{r}$ solution.

\section{Diagnostic method}

In the current work experimental radial electric field profiles are inferred from measurements of the perpendicular velocity $\left(u_{\perp}\right)[28$, as provided by the x-ray imaging crystal spectrometer (XICS) diagnostic. To derive the radial electric field from the flux-surfaceaveraged perpendicular flow velocity $\left(U_{\perp}\right)$ the radial force balance equation can be used: $\left\langle E_{r}\right\rangle=\left(1 / e n_{I} Z_{I}\right) \partial p_{I} / \partial \rho\langle|\nabla \rho|\rangle-\left\langle u_{\perp} B\right\rangle$, where $p_{I}, n_{I}$ and $Z_{I}$ denote the pressure, density and charge of the ion species being measured. The pressure gradient term is small for $A r^{16+}$ where this term is weighted by $1 / Z_{I}=1 / 16$ and has been neglected in the current calculations. 
The XICS diagnostic is based on spectroscopic analysis of emission from highly charged argon impurities that are seeded into the plasma in trace amounts [38. The XICS system records a 1D image of line-integrated spectra. The local plasma parameters are then found by utilizing tomographic inversion techniques with a known vMEc equilibrium [12, 39]. Standard Doppler spectroscopy techniques are used to extract information from the recorded spectra: Ion temperatures $\left(T_{i}\right)$ are found from the line widths, electron temperatures $\left(T_{e}\right)$ from line ratios, plasma flow $(u)$ from the line shifts, and impurity densities $\left(n_{A r^{15+/ 16+/ 17+}}\right)$ from the line amplitudes[38]. The viewing geometry of the XICS system, which is close to lying in a poloidal plane, is primarily sensitive to the component of the velocity that is perpendicular to the magnetic field $\left(u_{\perp}\right)$ (see Ref. 28, Fig. 1).

Detailed descriptions of the XICS diagnostic can be found in Ref. 27] and the diagnostic concept has been explained by Bitter et al. in Ref. 38. The procedure for determining the $E_{r}$ profile from the line-integrated XICS measurements is described in Ref. 28 and Ref. 12. For the current work two improvements have been made to the procedure that result in slight improvements in the quality of the inverted profiles.

The first change is that in the inversion of the emissivity (which is the first step in the inversion process) a simple parameterization has been added that allows the emissivity to vary poloidally on a flux surface. It has been found that in many W7-X plasmas, particularly before boronization, the measured $A r^{16+}$ emissivity was not completely consistent with the assumption of constant density/emissivity on a flux surface. The source of this asymmetry is not yet known, however a possible explanations are that it is due to $A r^{17+}$ charge exchange with neutral hydrogen [40] or due to variations of the plasma potential on a flux surface (known as the neoclassical $\phi_{1}$ term) [41].

The second change is an empirical wavelength correction that has been added on top of the standard geometry/wavelength calibration. This correction has been implemented as a quadratic offset from the nominal zero wavelength across the detector, and is determined by using a reference plasma where a long period of time averaging can be used to provide improved signal to noise for the calibration. The need for this empirical correction is likely due to subtle effects of spherical aberration or small errors in the characterization of the hardware dimensions.

\section{Sensitivity}

For the results discussed in Fig 3 and Fig 4 the simplifying assumption of $Z_{\text {eff }}=1$ and the use of a vacuum (zero plasma pressure) vMEc equilibrium were used. In this section the sensitivity of the neoclassical calculations to these assumptions is examined, along with a study on the sensitivity to the temperature and density profiles.

To examine the sensitivity to $Z_{\text {eff }}$ several SFINCS runs were done in which a carbon $\left(C^{6+}\right)$ profile was added so as to produce a constant $Z_{\text {eff }}$ profile across the plasmas. In these runs the electron density was kept unchanged, but the hydrogen density was reduced to maintain charge neutrality. The results of these scans are shown for two times in Fig 8 (a) and (b). These figures show only a small reduction in the magnitude of $E_{r}$ with increasing $Z_{\text {eff }}$ and an almost negligible change in the total NC energy flux (except at an 

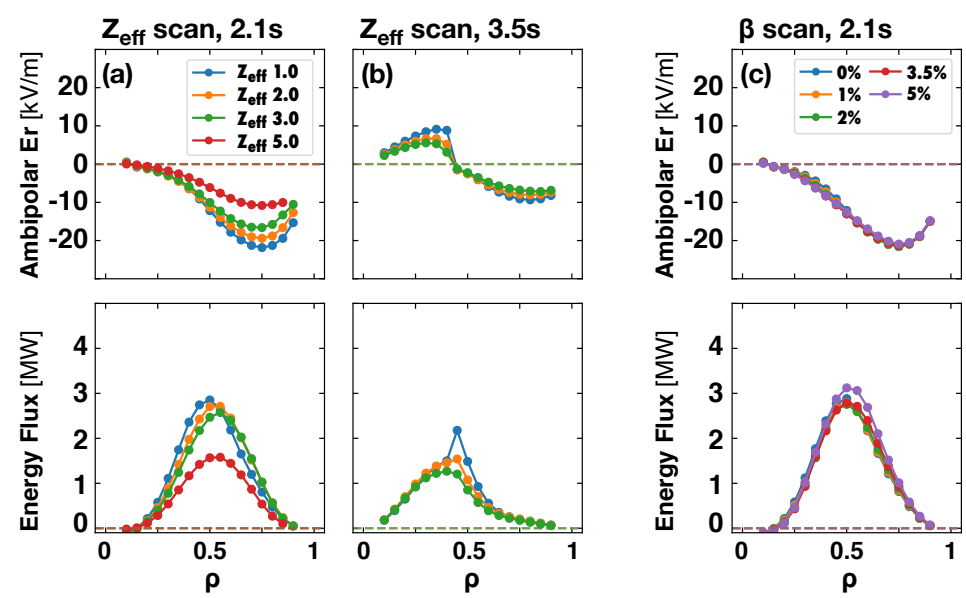

FIG. 8: Sensitivity of SFInCS calculations to $Z_{\text {eff }}$ and finite pressure equilibria. In figures (a) and (b) several SFINCS calculations are shown in which a carbon $\left(C^{6+}\right)$ profile has been added so as to produce a flat $Z_{\text {eff }}$ profile with the values shown. In figure (c) calculations were done with several different finite pressure VMEC equilibria. In this scan only the equilibrium was modified to allow a dedicated study of the geometrical effects; the diagnostic profiles and mapping to flux surface remain unchanged.

extreme value of $Z_{\text {eff }}=5$ ).

The second sensitivity scan, shown in Fig.8 (c) investigates the effect of finite pressure equilibria on the $\mathrm{NC}$ calculations. For this scan various equilibria were created using pressure profiles that are linear in the vMEc flux coordinate $(s)$ and result in volume averaged beta values ranging between $1 \%$ and $5 \%$. The VMEC calculations were done in free boundary mode with the total enclosed flux and the iota profile adjusted to maintain the minor radius and a vanishing toroidal current density. These equilibria were then used instead of the vacuum equilibrium during the SFINCS calculations. This scan goes well beyond the actual value of beta found from the kinetic profiles, which was around $1.3 \%$ at $t=2.2 \mathrm{~s}$. For this scan only the geometrical effects on the NC calculation were considered; in particular no changes were made in the mapping of the temperature and density profiles to flux space, nor were the profiles adjusted to match the scanned vMEC pressure profile. From the results of this scan it can be concluded that the NC calculations are almost completely insensitive to changes in the equilibrium due to finite beta effect. It must be noted that this finding should not be taken as a general result and is only valid for the standard magnetic configuration (EIM) [42, and for the specific profiles used in the calculation. A larger effect would be expected for other magnetic configurations and would also be expected if the temperature and density profiles had stronger gradients in the core.

The final set of scans are aimed at understanding the sensitivity of the NC results to changes in the temperature and density profiles. While the errors in the profile measurements due to photon statistics (random errors) are well characterized (shown as error bars in Fig.3), systematic errors are more difficult to quantify, for example due to diagnostic calibration, approximations in the analysis, or incorrect mapping to flux surface coordinates. The scans shown here give an indication of how any such errors might affect the final NC results. For this set of scans the temperature and density profiles were indi- 

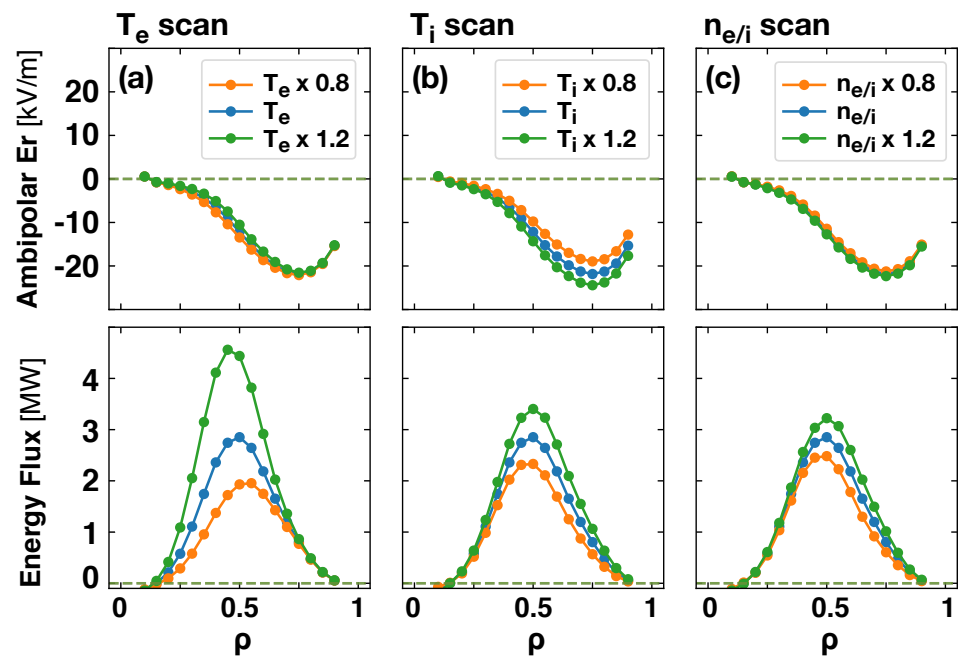

FIG. 9: Sensitivity of SFINCS calculations to scaling of the temperature and pressure profiles. For each set of plots the the ambipolar $E_{r}$ and total energy flux is calculated with the specified profile scaled by 80\% and 120\%, while the other profiles are held fixed. In (a) the electron temperature is scaled, in (b) the ion temperature is scaled, and in (c) both the ion and electron densities are scaled. These plots are only meant as an exploration of sensitivity and do not correspond to experimental error bars.

vidually scaled up and down by $20 \%$ and the NC parameters recalculated. The results of these scans are shown in Fig.9. These scans are only meant to illustrate profile sensitivity; the scan range was chosen arbitrarily and should not be interpreted as error bars. The range of $20 \%$ is larger than any expected systematic errors, especially in the case of the $T_{e}$ which has been cross validated between several diagnostics [43, 44] and is expected to have small systematic errors.

This set of sensitivity scans shows that the NC results detailed in this paper are robust against the assumptions used and any systematic errors in the profile measurements. This is particularly true for the overall magnitude and shape of the $\mathrm{NC}$ energy flux and $E_{r}$ profiles.

\section{Discussion}

Measurements of the core radial electric field $\left(E_{r}\right)$ have confirmed that ion-root conditions (negative $E_{r}$ in the plasma core) have been achieved in W7-X with high-density pelletfueled plasmas and central ECRH heating. The shape and evolution of the measured $E_{r}$ profiles agree well with the neoclassical ambipolar $E_{r}$ as predicted by the code SFINCS 32. over a wide range of temperatures and densities. In terms of magnitude, the measured $E_{r}$ profiles are generally in agreement to within better than a factor of two. Several other plasmas have also been examined to better understand parameter dependencies and behavior of the $E_{r}$ profile, including a study of profile shaping through off-axis heating and a comparison between two magnetic configurations.

There are several possible explanations for mismatches in magnitude between the XICS measurements and the NC predictions that are seen in the $E_{r}$ profile comparisons, 
particularly in the core. The most likely cause is errors in the experimental $E_{r}$ profiles due to systematic errors in the diagnostic calibration. The final analyzed profiles are quite sensitive to errors in the wavelength calibration of the diagnostic, which are difficult to characterize and likely lead to uncertainty on the order of the discrepancies that are seen. It is important to note that no attempt has been made to adjust the calibration of the XICS diagnostic in order to improve agreement with the NC results. Other possible sources for systematic errors in the experimental $E_{r}$ profiles are inaccuracies inherent in the tomographic inversion procedure and the impact of parallel velocity on the diagnostic measurements (expected to be small).

The general agreement between $\mathrm{NC}$ and measured $E_{r}$ profiles (given the current experimental uncertainties) provides confidence in the validity of neoclassical calculations over a wide range of plasma parameters and in particular in high-density ion-root conditions. This enables initial studies on the role of NC in the optimized high-density regime of W7-X. In addition this agreement, in particular in the case of $E_{r}$ profile shape, evolution and parameter dependence, supports the conclusion that turbulent transport in $\mathrm{W} 7-\mathrm{X}$ is intrinsically ambipolar.

These comparisons between neoclassical and experimental $E_{r}$ profiles also have important implications for the success of the W7-X design in reducing neoclassical transport. The W7-X design was, in part, based on the calculation of neoclassical transport using calculations similar to those provided here by sfincs. Previous work has validated the use of these codes for both $E_{r}$ and bootstrap current in low density operation in W7-X and compared different NC codes (see Ref. 28 and Ref. 44). The current work now provides an experimental indication that these calculation are also applicable to high-density ion-root operation, which is near the target point of the NC optimization. Finally these measurements of $E_{r}$ in W7-X also support the use of these predictive tools in the future design of NC optimized stellarators.

Research supported by the U.S. DOE under Contract No. DE-AC02-09CH11466 with Princeton University. This work has been carried out within the framework of the EUROfusion Consortium and has received funding from the Euratom research and training programme 2014-2019 under grant agreement No 633053. The views and opinions expressed herein do not necessarily reflect those of the European Commission.

\section{References}

[1] NÜHRENBERG, J. et al., Physics Letters A 114 (1986) 129.

[2] BEIDLER, C. et al., Fusion Technology 17 (1990) 148.

[3] MAASSBERG, H. et al., Physics of Fluids B: Plasma Physics 5 (1993) 3728.

[4] WARMER, F. et al., Plasma Physics and Controlled Fusion 58 (2016) 074006.

[5] SUGAMA, H. et al., Physics of Plasmas 3 (1996) 2379.

[6] HELANDER, P. et al., Phys. Rev. Lett. 101 (2008) 145003.

[7] MAASSBERG, H. et al., Physics of Plasmas 16 (2009).

[8] DINKLAGE, A. et al., 43rd EPS Conference on Plasma Physics (2016) O2.10\%. 
[9] YOKOYAMA, M. et al., Nuclear Fusion 47 (2007) 1213.

[10] DINKLAGE, A. et al., Nuclear Fusion 53 (2013) 063022.

[11] IDA, K. et al., Nuclear Fusion 45 (2005) 391.

[12] PABLANT, N. A. et al., Plasma Physics and Controlled Fusion 58 (2016) 045004.

[13] BALDZUHN, J. et al., Plasma Physics and Controlled Fusion 40 (1998) $96 \%$.

[14] EHMLER, H. et al., Nuclear Fusion 43 (2003) L11.

[15] GUTIÉRREZ-TAPIA, C. et al., Plasma Physics and Controlled Fusion 57 (2015) 115004.

[16] KUMAR, S. et al., Nuclear Fusion 57 (2017) 036030.

[17] BRIESEMEISTER, A. et al., Plasma Physics and Controlled Fusion 55 (2013) 014002.

[18] MINAMI, T. et al., 42rd EPS Conference on Plasma Physics (2015) P5.103.

[19] IDA, K. et al., Physics of Fluids B: Plasma Physics (1989-1993) 3 (1991) 515.

[20] KENMOCHI, N. et al., 42rd EPS Conference on Plasma Physics (2015) P5.131.

[21] PEDERSEN, T. S. et al., Plasma Physics and Controlled Fusion 61 (2018) 014035.

[22] BOZHENKOV, S. et al., Proceedings of the 27th IAEA Fusion Energy Conference (2018) $E X / P 8$.

[23] BOZHENKOV, S. et al., Nuclear Fusion (2019), submitted for publication.

[24] KLINGER, T. et al., Nuclear Fusion (2019), accepted for publication.

[25] VELASCO, J. L. et al., Plasma Physics and Controlled Fusion 55 (2013) 124044.

[26] WOLF, R. C. et al., Physics of Plasmas (2019), submitted for publication.

[27] LANGENBERG, A. et al., Review of Scientific Instruments 89 (2018) $10 G 101$.

[28] PABLANT, N. A. et al., Physics of Plasmas 25 (2018) 022508.

[29] PASCH, E. et al., 43rd EPS Conference on Plasma Physics (2016) P4.016.

[30] BRUNNER, K. et al., Journal of Instrumentation 13 (2018) P09002.

[31] VAN RIJ, W. I. et al., Physics of Fluids B 1 (1989) 563.

[32] LANDREMAN, M. et al., Physics of Plasmas 21 (2014).

[33] KRYCHOWIAK, M. et al., Review of Scientific Instruments 87 (2016) 11D304.

[34] HIRSHMAN, S. et al., Computer Physics Communications 43 (1986) 143.

[35] PAVONE, A. et al., Journal of Instrumentation (2019), submitted for publication.

[36] RAHBARNIA, K. et al., Nuclear Fusion 58 (2018) 096010.

[37] MCNEELY, P. et al., Fusion Engineering and Design 88 (2013) 1034, Proceedings of the 27th Symposium On Fusion Technology (SOFT-27); Liège, Belgium, September 24-28, 2012.

[38] BITTER, M. et al., Review of Scientific Instruments 81 (2010) 10 E328.

[39] PABLANT, N. A. et al., Review of Scientific Instruments 85 (2014) 11E424.

[40] BEIERSDORFER, P. et al., Phys. Rev. A 72 (2005) 032725.

[41] GARCÍA-REGAÑA, J. et al., Nuclear Fusion 57 (2017) 056004.

[42] GEIGER, J. et al., Plasma Physics and Controlled Fusion 57 (2014) 014004.

[43] WOLF, R. C. et al., Nuclear Fusion (2017).

[44] DINKLAGE, A. et al., Nature Physics (2018). 